Results: 78 studies ( $n=5,639$ participants) met the inclusion criteria. There was a high risk of bias on blinding and most trials had small sample size $(n<50)$. While multidisciplinary treatment (MDT) was the best for improving pain $[-1.28$ $(-1.84,-0.72)]$, sleep $[-1.14(-2.38,0.07)]$ and depression $[-1.20(-1.99,-0.46)]$, balneotherapy and exercise were the most effective treatments for FIQ [-1.06 $(1.51,-0.61)]$ and fatigue $[-0.75(-1.35,-0.25)]$, respectively (Figure 2$)$.

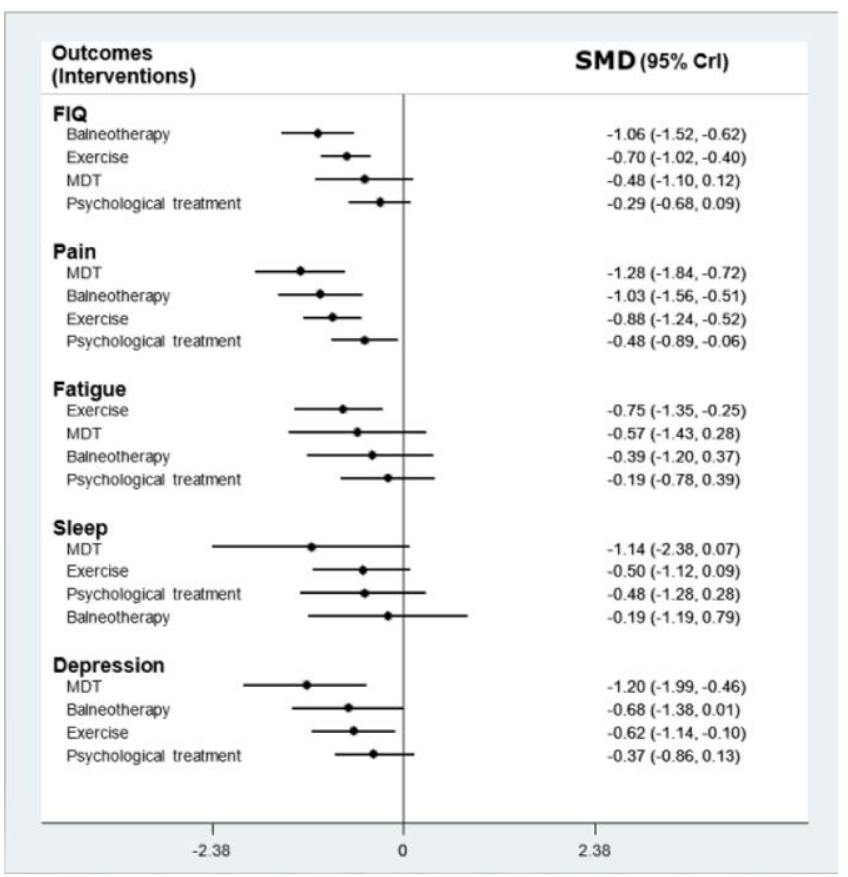

Figure 2. Standardised mean difference (SMD) versus usual care in descending order for different outcomes

Data from 47 exercise trials ( $n=3,271$ participants) were analysed to examine comparative efficacy of different exercise types. Strengthening showed the greatest benefits for FIQ $[-0.76(-1.39,-0.15)]$, pain $[-0.94(-1.58,-0.29)]$ and depression $[-0.83(-1.53,-0.14)]$, whereas aerobic exercise was the best for fatigue $[-0.98(-2.33,0.18)]$ and sleep $[-0.96(-2.08,0.13)]$ (Table 1).

Table 1. Relative effect size between types of exercises

\begin{tabular}{llllll}
\hline FIQ & & & & & \\
\hline Aerobic & -0.58 & -0.09 & 0.18 & 0.12 & -0.57 \\
& $(-1.13,-0.03)$ & $(-0.55,0.36)$ & $(-0.44,0.80)$ & $(-0.36,0.57)$ & $(-0.95,-0.24)$ \\
-0.60 & Flexibility & 0.49 & 0.76 & 0.70 & 0.004 \\
$(-1.36,0.18)$ & & $(-0.23,1.20)$ & $(-0.07,1.58)$ & $(-0.04,1.41)$ & $(-0.67,0.64)$ \\
-0.10 & 0.49 & Mind-body & 0.27 & 0.20 & -0.49 \\
$(-0.74,0.53)$ & $(-0.50,1.49)$ & & $(-0.37,0.91)$ & $(-0.24,0.65)$ & $\mathbf{( - 0 . 8 5 , - 0 . 1 5 )}$ \\
0.05 & 0.65 & 0.16 & Mixed & -0.06 & $-\mathbf{0 . 6 9}$ \\
$(-0.59,0.70)$ & $(-0.35,1.63)$ & $(-0.53,0.85)$ & & $(-0.73,0.59)$ & $\mathbf{( - 1 . 0 6 , - 0 . 3 4 )}$ \\
0.21 & 0.80 & 0.31 & -0.06 & Strengthening & -0.76 \\
$(-0.42,0.84)$ & $(-0.19,1.79)$ & $(-0.41,1.02)$ & $(-0.73,0.59)$ & & $(-1.39,-0.15)$ \\
-0.73 & -0.13 & -0.62 & -0.78 & -0.94 & Usual care \\
$(-1.16,-0.30)$ & $(-1.02,0.74)$ & $(-1.15,-0.11)$ & $(-1.31,-0.26)$ & $(-1.58,-0.29)$ & \\
Pain & & & & &
\end{tabular}

Data are standard mean difference ( $95 \%$ credible intervel) between exercises, pairwised from the top left to the bottom right. The negative value indicates that the first exercise is more effective than the second exercise. For example, aerobic is better than flexibility for FIQ -0.58 $(-1.13,-0.13)$, but not pain $-0.60(-1.36,0.18)$.

Conclusion: Several non-pharmacological interventions are beneficial for FM. However, the effect size varies between interventions and outcomes. All types of exercises are effective for FIQ and pain apart from flexibility exercise. The results of this study may be used to guide the selection of the most effective non-pharmacological interventions according to the predominant symptom in individual patients.

References:

[1] Macfarlane GJ et al. Ann Rheum Dis 2017;76(2):3-8-28.

Disclosure of Interests: : None declared

DOI: 10.1136/annrheumdis-2020-eular.803

\section{THU0462 CHARACTERIZATION OF PATIENTS WITH FIBROMYALGIA AFFECTS WITH OR WITHOUT JOINT HYPERLAXITY SYNDROME}

L. López-Núñez ${ }^{1,2}$, J. Blanch ${ }^{1}$, M. Ciria Recasens ${ }^{1}$, M. J. Herrero Gascón ${ }^{1}$, A. Juan-Mas ${ }^{3}$, J. Carbonell Abelló ${ }^{1}{ }^{1}$ Hospital del Mar, Reumatología, Barcelona, Spain; ${ }^{2}$ Hospital Quiron Salud, Reumatologia, Mallorca, Spain $;{ }^{3}$ Hospital Son Llátzer, Palma de Mallorca, Spain

Background: The prevalence of joint hypermobility $(\mathrm{JH})$ and Joint Hypermobility Syndrome (JHS) in patients with fibromyalgia (FM) is considerable and is much more than can be explained at random(1). Some authors propose that FM and JHS share a common pathophysiological mechanism is some patients. Currently it is accepted that Ehlers-Danlos Syndrome Hypermobility subtype (EDSh) and JHS are the same entity. We regard the subgroup of FM patients with JHS a different subtype of FM, even phenotypically similar to EDSh.

Objectives: Determine the possible differences between both groups according to their body composition, bone metabolism and clinical findings.

Methods: Our study is observational, descriptive, transverse cohort study in which we included 86 women with fibromyalgia recruited at the Fibromyalgia and Chronic Fatigue Unit at Parc Salut-Mar in Barcelona, Spain. The patients were grouped according to the presence or absence of JHS, following the Brighton Criteria. Diverse clinical data was collected: Pain Visual Analogue Scale (PVAS), time from pain onset, time from diagnosis, somatic symptoms, state of mind, presence of a FM trigger, concurrent medication, anxiety, quality of life, disease impact, anthropometric data, Bioelectrical Impedance Analysis (BIA), bone density test (BMD) and bone metabolism data in blood samples.

Results: 51 patients were included in the FM group and 35 patients in the FM-JHS group. We did not find differences between groups PVAS; time from pain onset; somatic symptoms using the Psychiatric Disorder and Somatic Pathology Scale (TOPYPS); nor Fibromyalgia Impact Questionnaire (FIQ). Both groups scored similarly on SF-36 Health Questionnaire. The use of opioids was more common in the FM group $(p<0,001)$. Anxiety disorder $(A n D)$ was present in a greater proportion of FM-JHS $(p<0,001)$. We found the Body Mass Index and Muscle Mass (MM) to be less in the FM-JHS group ( $p=0.001$ and $p=0.008$, respectively). Obesity and fat mass (FatM) were more frequent in the FM group. The FatM and less MM correlated with less quality of life on the SF-36 scale. There was less bone mass (BM) in the FM-JHS group $(p=0.005)$. We found an inverse correlation between the Beighton score and the MM and BM in the FM-JHS group. The FM-JHS group also had less bone mineral density (BMD) at total hip DXA, with significant differences $p=0.038$. The BM by Bioelectrical Impedance Analysis (BIA) had a positive correlation on the BMD by DXA. The optimum point, capable of distinguishing between normal DXA and osteopenia/ osteoporosis was $2,325 \mathrm{~kg}$ with a specificity of $86 \%$ and sensibility of $52 \%$. Vitamin D deficiency/insufficiency was found in $62 / 84(73,8 \%)$ without significant differences between groups $(p>0,05)$

Conclusion: Our work revealed that FM patients with JHS are different from FM without JHS, by manifesting differences in certain clinical, anthropometric, and bone metabolism features.

\section{References:}

[1] Lai S, Goldman JA, Child AH, Engel A, Lamm SH. Fibromyalgia, hypermobility, and breast implants. J Rheumatol. 2000;27(9):2237-41.

[2] Malfait, F., Francomano, C., Byers, P., Belmont, J., Berglund, B., Black, J., ... \& Castori, M. (2017, March). The 2017 international classification of the Ehlers-Danlos syndromes. In American Journal of Medical Genetics Part C: Seminars in Medical Genetics (Vol. 175, No. 1, pp. 8-26).

Disclosure of Interests: : None declared

DOI: 10.1136/annrheumdis-2020-eular.4115

\section{THU0463 EFFICACY AND SAFETY OF NERIDRONATE IN BONE EDEMA SYNDROME}

A. M. Lurati ${ }^{1}$, A. Laria ${ }^{1}$, P. Faggioli ${ }^{2}$, L. Castelnovo ${ }^{2}$, A. Tamburello ${ }^{2}$, A. Mazzone ${ }^{2} .{ }^{1}$ Rheumatology Unit Fornaroli Hospital, Magenta, Italy; ${ }^{2}$ Internal Medicine Unit OSpedale Civile, Legnano, Italy

Background: Bone Marrow Edema Syndrome (BMES) is a severely disabling pain syndrome without a definite treatment and refers to transient clinical conditions with unknown pathogenic mechanism, such as transient osteoporosis of the hip $(\mathrm{TOH})$, regional migratory osteoporosis (RMO), and reflex sympathetic dystrophy (RSD). Magnetic resonance imaging is used for the early diagnosis and monitoring the progression of the disease. Early differentiation from other aggressive conditions with long-term sequelae is essential in order to avoid unnecessary treatment.

Objectives: Aim of this monocentric trial was to test the efficacy and the safety of the amino-bisphosphonate neridronate in patients with BMES administered in two different regimens. 
Methods: 192 patients with BMES secondary to osteoarthritis localized to knee, hip, wrist or foot were randomly assigned to I.V. infusion of $100 \mathrm{mg}$ neridronate given four times over 10 days (Group A, 72 subjects) or alternatively to I.V. infusions of $100 \mathrm{mg}$ every 21 days over 3 months (Group B, 120 subjects). At baseline and after 180 days we performed an MRI. We assessed a 0-100 mm pain VAS in each patient, too. Outcomes were to evaluate the MRI changes and the VAS changes. A control group (35 patients) was enrolled too, treated conservatively with NSAIDs and articular rest. Results: we observed a significant improvement in MRI with the resolution of bone marrow lesions present at the baseline $(p<0.01)$, without a significant difference between Group A and Group B. Visual analogue scale (VAS) score decreased significantly during the study in both groups $(p<0.05)$ without a significant difference between the two treatment groups $(p>0.1)$. Both groups showed a significant clinical and radiologic improvement compared with control group $(p<0.001)$.

Conclusion: In patients with BMES, the infusions of neridronate $100 \mathrm{mg}$ every 21 days over 3 months or alternately every 3 days over 10 days are associated with clinically relevant and persistent benefits without significant differences between the two treatment-schedules. These results provide conclusive evidence that the use of bisphosphonates, at appropriate doses, is the treatment of choice BMES.

Disclosure of Interests: : None declared

DOI: 10.1136/annrheumdis-2020-eular.4353

\section{THU0464 USE OF BENZODIAZEPINES AND ANTIDEPRESSANTS IN PATIENTS WHO ATTEND A RHEUMATOLOGY CLINIC}

M. P. Martínez-Vidal ${ }^{1}$, A. Alvarez de Cienfuegos ${ }^{2}$, V. Jovani ${ }^{3}$, R. MartínDomenech $^{4}$, M. T. Pedraz-Penalva ${ }^{4}$, P. Bernabéu ${ }^{3}$, J. R. Noguera-Pons ${ }^{5}$ ${ }^{1}$ Hospital Universitario San Juan de Alicante, Alicante, Spain; ${ }^{2}$ Hospital Vega Baja, Orihuela, Spain; ${ }^{3}$ Hospital General Universitario de Alicante, Alicante, Spain; ${ }^{4}$ Hospital General Universitario de Elda, Elda, Spain; ${ }^{5}$ Hospital General Universitario de Elche, Elche, Spain

Background: During the last decades, anxiolytics and antidepressants (ADP) have been among the most prescribed therapies in all developed countries (1). In Spain a prevalence of use of $11.4 \%$ was communicated (2), slightly over the European average $(3,4)$. They have potential addiction problems and side effects. Objectives: The objective of this study was to evaluate the prevalence of anxiolytics and antidepressants among patients who attend a Rheumatology consult, as well as the indication for them.

Methods: Patients who were referred for the first time to the Rheumatology consult were included. Demographical data, reason for referral and final diagnosis were recorded. Regarding the treatment with ADP or/and benzodiazepines (BDZ), their duration and the indication for the prescription were recorded. Sample size was estimated for a $0.05 \%$ alpha risk. Descriptive, univariate and multivariate analyses (ANOVA) were performed in order to study the prevalence of these treatments, and their associations with demographical or clinical characteristics. The study was approved by the Hospital Universitario de Elche Ethics Committee. Results: 350 patients were included (women $77.1 \%$, men $22.9 \%$ ), mean age 58.1 yo. $40 \%$ were occupied and $31.4 \%$ were unemployed. The majority were married or lived with a couple $(71.4 \%)$. Most of them had been referred for musculoskeletal pain (73.4\%). More than a third (39.4\%) were on BZD and/or ADP: 107 patients were on BZD (30.6\%), 68 were on ADP (19.4\%), and $47(13.4 \%)$ were on both. The most frequent reasons for their prescription were anxiety, depression and insomnia. The final diagnosis in the clinic was a non-inflammatory condition in $53.1 \%$, and inflammatory in $18 \%$. In the univariate analyses, the use of BZD/ADP was not associated with civil status, but it was associated with female sex $(p<0.001)$, unemployment $(p<0.001)$ and non-inflammatory final diagnosis $(p<0.001)$. In the multivariate analyses, the use of BZD and/or ADP was associated with female sex $(p=0.002$ [RR 3.4, Cl 95\% 1.6-7.4]) and non-inflammatory final diagnosis, specifically fibromyalgia ( $p=0.007$ [RR 16.1, Cl 95\% 2.2-120.7]). Conclusion: The use of anxiolytics and antidepressants is frequent in the patients referred to the Rheumatology clinic, and it's associated to female sex and non-inflammatory conditions, over all fibromyalgia.

References:

[1] Lagnaoui R, Depont F, Fourrier A, Abouelfath A, Bégaud B, Verdoux H, et al. Patterns and correlates of benzodiazepine use in the French general population. Eur J Clin Pharmacol. 2004;60:523-9.

[2] Ministerio de Sanidad, Servicios Sociales e Igualdad. Secretaría de Estado de Servicios Sociales e Igualdad. Delegación del Gobierno para el Plan Nacional sobre Droga. Encuesta sobre alcohol y drogas en población general en España: EDADES 2011-2012. 2013. Disponible en: http://www.pnsd. msc.es/Categoria2/observa/pdf/EDADES2011.pdf

[3] Simó Miñana J. Utilización de medicamentos en España y en Europa. Aten Primaria. 2012; 44:335-47.

[4] Khong TP, de Vries F, Goldenberg JS, Klungel OH, Robinson NJ, Ibáñez L, Petri $\mathrm{H}$. Potential impact of benzodiazepine use on the rate of hip fractures in five large European countries and the Unite d States Calcif Tissue Int. 2012;91:24-31.
Disclosure of Interests: : None declared

DOI: 10.1136/annrheumdis-2020-eular.610

\begin{tabular}{|l|l|}
\hline THU0465 & SERUM LEVEL OF ADRENOCORTICOTROPHIC \\
HORMONE IS A CONTRIBUTING HORMONE OF \\
METABOLIC SYNDROME IN NEWLY DIAGNOSED \\
FIBROMYALGIA
\end{tabular}

T. Merza Mohammad ${ }^{1}, \mathrm{M}$. Alnimer ${ }^{2} .{ }^{1}$ Hawler Medical University, College of Pharmacy, Erbil, Iraq; ' Ja'affar Sadiq University, Bagdad, Iraq, Baghdad, Iraq

Background: Evidence of components of metabolic syndrome including. Obesity dyslipidemia, abnormal glucose tolerance rate and hypertension are associated with fibromyalgia. Adrenocorticotrophic hormone (ACTH) is reported to be significantly higher in fibromyalgia patients, and it causes obesity, high blood pressure. Objectives: This study aimed to assess the serum level of ACTH as a contributing as well as a discriminator hormone in newly diagnosed fibromyalgia women presented with variable components of metabolic syndrome.

Methods: This cross-sectional study comprised 100 women with newly diagnosis fibromyalgia and 30 apparent healthy women served as control from Kurdistan region-Iraq. Clinical data including the score of fibromyalgia impact questionnaire-revised (FIQR), tender point, body mass index, waist circumference, blood pressure and fasting serum levels of glucose and lipid profile, and ACTH level. The score of metabolic syndrome was calculated using the formula:

$$
\begin{aligned}
& \frac{2 \times \text { waist }}{\text { height }}+\frac{\text { serumglucose }}{5.6}+\frac{\text { Serumtriglyceride }}{1.7} \\
& +\frac{\text { Systolic blood pressure }}{130}-\frac{\text { serum highdensity lipoprotein }}{1.02 \text { or } 1.28 \text { (maleor female) }}
\end{aligned}
$$

Results: Compared to the controls, the Fibromyalgia women displayed significantly higher values of waist circumference $(88.9 \pm 5.7 \mathrm{~cm}$ versus $87.1 \pm$ $2.7 \mathrm{~cm}, \mathrm{p}=0.019)$, systolic blood pressure $(136.1 \pm 13.5 \mathrm{mmHg}$ versus $131.4 \pm 7.1$ $\mathrm{p}=0.014)$, metabolic syndrome score $(3.10 \pm 0.25$ versus $3.03 \pm 0.19, p=0.039)$, and serum ACTH levels $(16.66 \pm 3.23 \mathrm{pg} / \mathrm{ml}$ versus $14.42 \pm 2.18 \mathrm{pg} / \mathrm{ml} . \mathrm{p}<0.001)$ Serum ACTH levels significantly and inversely correlated with the total score of the FIQR $(r=-0.320 . p=0.001)$ and number of tender points $(r=-0.374, p<0.001)$. Metabolic syndrome score is significantly and inversely correlated with the total FIQR score $(r=-0.296, p=0.003)$. Multivariable regression analysis using showed that serum level of ACTH is a significant ( $p=$ predictor of $19.7 \%$ of fibromyalgia patients (Figure 1$)$, and it is a significant $(p=0.007)$ discriminator of tender points as the area under the curve is $0.325\left(_{95 \%}\right.$ C.I.: $0.212-0.438$ ) (Figure 2).

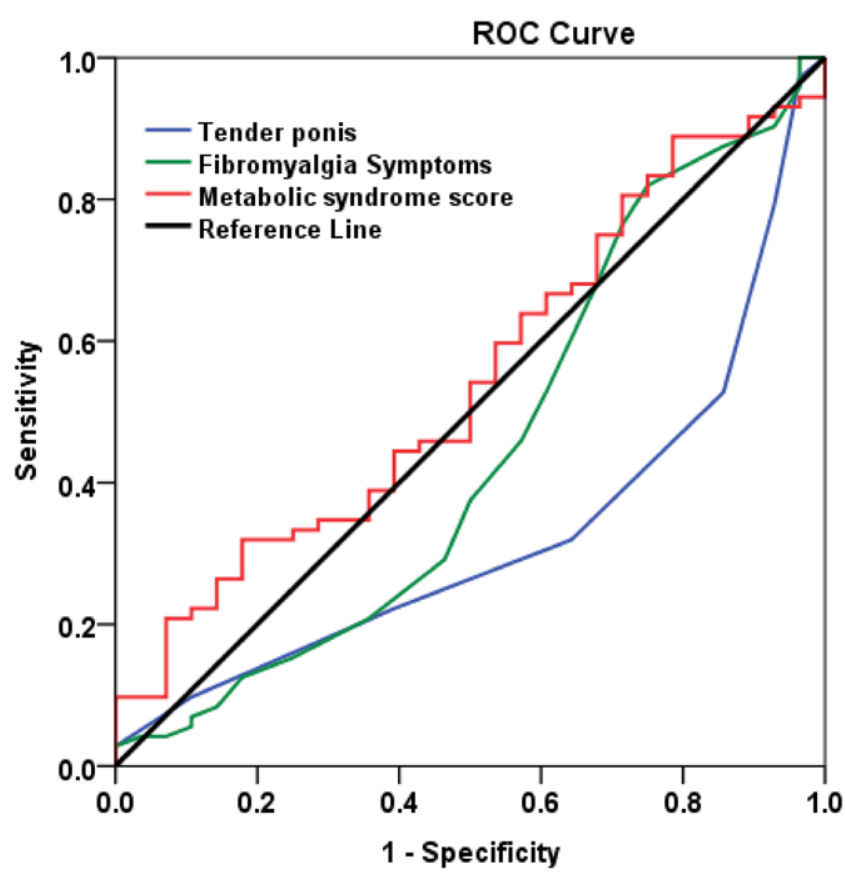

Diagonal segments are produced by ties.

Figure 2. serum level of ACTH (cutoff level $\geq 14.5 \mathrm{pg} / \mathrm{ml}$, sensitivity $=72 \%$, specificity $=50 \%$ ) as a discriminator of the tender points (AUC ${ }_{95 \%} \mathrm{cl}: 0.325[0.212-0.438], p=0.007$ ), score of 